\section{Validez y confiabilidad de las escalas de evaluación funcional en pacientes críticamente enfermos. Revisión sistemática}

\author{
MARCELA LIBUY H. ${ }^{1, \mathrm{a}}$, PAOLA SZITA C. ${ }^{1, \mathrm{a}}$, \\ JUAN HERMOSILLA P. ${ }^{2,3}$, DANIEL ARELLANO S., 4,6,a, \\ IVÁN RODRÍGUEZ-NÚÑEZ1,5,6,a,b, CLAUDIO BÁEZ R. .,5,a
}

\section{Validity of scales for the functional assessment of critically ill patients}

The decrease in mortality in critical patient units led to an increase in intensive care unit acquired weakness (ICUAW), which significantly affects the functional performance and quality of life of patients. There are several scales that measure functionality in critical patients. The aim of this systematic review is to determine the criterion validity and reliability of the scales that evaluate functionality in critically ill adult patients. We considered studies in critically ill adult subjects of both genders that determined the psychometric properties of the scales that evaluate functionality. Six minutes' walk test (6MWT), timed up and go (TUG), the Medical Research Council sum score (MRC-SS), grip strength, discharge destination and need for rehabilitation at discharge were considered as gold standards. Three scales were identified: PFIT-s, Perme MS, and DEMMI. PFIT-s has a positive correlation with 6MWT, MRC-SS and grip strength, and a negative correlation with TUG. It also predicts the need for rehabilitation at discharge and discharge to the home. DEMMI has a positive correlation with $M R C-S S$. the interobserver reliability was evaluated in three articles, demonstrating an almost perfect association. The intraobserver agreement was considered good in one report. With this information, it is not possible to determine which is the instrument with better measurement properties.

(Rev Med Chile 2017; 145: 1137-1144)

Key words: Critical Care Outcomes; Intensive Care Units; Mobility Limitation; Recovery of Function; Validation Studies.

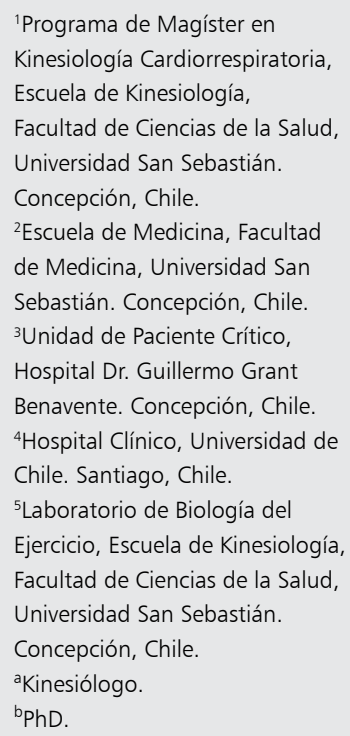

Financiamiento: Este estudio fue financiado por la Escuela de Kinesiología de la Universidad San Sebastián, Chile.

Los autores no declaran conflicto de interés.

Recibido el 7 de marzo de 2017, aceptado el 28 septiembre de 2017.

Correspondencia a:

Claudio Báez Rojas. Lientur 1457, Universidad San Sebastián, Concepción. claudio.baez@uss.cl
E 1 aumento de la supervivencia, en las unidades de paciente crítico (UPC) es una de los factores asociados al incremento de la debilidad adquirida en UCI (DAUCI). Este cuadro clínico se define como un síndrome de debilidad generalizada de las extremidades, para el cual no existe una explicación alternativa que no sea la propia enfermedad crítica del paciente ${ }^{2}$. Los principales factores de riesgo para el desarrollo de la DAUCI son el reposo prolongado en cama, la hiperglicemia, la sepsis, el uso de corticoesteroides y bloqueadores neuromusculares ${ }^{1,3-12}$. Fisiopatológicamente, estos factores se asocian con un desbalance entre la síntesis y degradación de proteínas musculares, asociado a necrosis de las miofibrillas, produciéndose una disminución rápida y significativa de la masa muscular que puede llegar a $15 \%$ los primeros 7 días de la enfermedad crítica, además de una disminución de la densidad mineral ósea ${ }^{13,14}$. 
Escalas de evaluación funcional en pacientes críticamente enfermos - M. Libuy et al

Entre los métodos diagnósticos utilizados para el establecimiento de la DAUCI se encuentra la electromiografía y la biopsia muscular, sin embargo, su alto costo y carácter invasivo han promovido el desarrollo de métodos diagnósticos simplificados como el Medical Research Council Sum Score (MRC-SS) cuyo puntaje se obtiene a partir de la cuantificación de la fuerza ejercida contra la resistencia manual aplicada por el evaluador, lo cual requiere la participación activa del paciente, por lo tanto debe ser aplicada posterior a la suspensión de la sedación ${ }^{15,16}$. Esto retarda significativamente el proceso diagnóstico dificultando el reconocimiento precoz de este síndrome durante la etapa de sedación del paciente. Es así como se han registrado tasas de incidencia variables que oscilan entre 25 y $75 \%{ }^{1,17,18}$.

Entre las principales consecuencias de la DAUCI se encuentran el retardo en el destete de ventilación mecánica $(\mathrm{VM})$ y aumento, tanto del tiempo de estadía en UPC, como de la morbimortalidad hospitalaria y de los $\operatorname{costos}^{19,20}$. Adicionalmente, la DAUCI se ha asociado al desacondicionamiento cardiorrespiratorio y muscular ${ }^{2,4,21,22}$, afectando significativamente el desempeño funcional $y$ calidad de vida de los pacientes; lo cual ha sido evidenciado hasta 5 años después del alta hospitalaria $^{5,6,22-27}$. Por este motivo, el desarrollo de instrumentos que permitan cuantificar de forma certera la magnitud del deterioro funcional en este tipo de pacientes es crucial para el desarrollo de estrategias terapéuticas apropiadas, dirigidas a atenuar el deterioro funcional asociado a la enfermedad crítica.

La evaluación de la funcionalidad considera diversos aspectos, tales como la comprensión de la tarea por parte del paciente, coordinación, procesamiento de la información visual, control motor central y activación de las vías motoras eferentes; todo esto además puede verse influenciado por el ambiente. Por lo tanto, es necesario el desarrollo de instrumentos que permitan determinar este constructo desde todas sus dimensiones. Es así como en los últimos años han surgido diversas escalas de evaluación funcional para pacientes $\operatorname{críticos}^{28,29,30-32}$, las cuales han mostrado validez y confiabilidad variables en los diferentes estudios clínicos. Por este motivo el presente estudio tiene por objetivo determinar la validez de criterio y confiabilidad de las escalas de funcionalidad para pacientes adultos críticamente enfermos.

\section{Material y Métodos}

\section{Diseño}

Revisión sistemática.

Criterios de elegibilidad de los artículos primarios

Se consideraron estudios en inglés y español dirigidos a determinar las propiedades psicométricas de las escalas de evaluación funcional para paciente crítico. Los estudios debían describir la asociación entre el puntaje de la escala de evaluación funcional y el resultado de un estándar de referencia relacionado con la capacidad funcional del paciente. Como estándar de referencia se consideró criterios frecuentemente utilizados en este tipo de estudios. Así para la validez de criterio concurrente se utilizó el resultado del Test de Marcha de 6 min (TM6), Timed Up and Go (TUG), Medical Research Council Sum Score (MRC-SS) y la fuerza de prensión. Por su parte, para la validez de criterio predictivo se consideraron destino al alta (domicilio o centro de cuidado agudo a largo plazo (CALP) y necesidad de rehabilitación al alta como criterios de referencia.

Como medida de asociación para la evaluación de la validez de criterio concurrente se consideró el coeficiente $r$ de Pearson o Rho de Spearman, para la validez de criterio predictivo se consideró la razón de probabilidades (OR) y el área bajo la curva ROC (AUC). Por su parte, como medida de asociación para la confiabilidad inter e intraobservador se consideró al coeficiente Kappa (k) para variables cualitativas y el coeficiente de correlación intraclase (CCI) para las variables cuantitativas.

\section{Sujetos de estudio}

Los estudios primarios debía incorporar pacientes críticamente enfermos, quienes son definidos como un enfermo cuya condición patológica afecta a uno o más sistemas poniendo en riesgo actual o potencial su vida y presenta condiciones de reversibilidad que hacen necesaria la aplicación impostergable de técnicas de monitorización, vigilancia, manejo y soporte vital avanzado ${ }^{33}$. Se consideraron estudios realizados en sujetos adultos de ambos géneros, críticamente enfermos, hospitalizados en una UPC médica o quirúrgica. Se excluyeron estudios realizados en pacientes con enfermedad neurológica, neuromuscular y/o traumatológica, así como también en individuos menores de 18 años sin análisis por subgrupo. 


\section{Metodología de evaluación de la funcionalidad}

Los estudios debían evaluar la funcionalidad mediante una escala de evaluación aplicada por un profesional con experiencia en el manejo de pacientes hospitalizados en una UPC. Al momento de la evaluación los sujetos de estudio debían encontrarse vigiles y cooperadores, en condiciones de estabilidad respiratoria y hemodinámica, y sin contraindicaciones para movilizarse en $\mathrm{cama}^{34}$.

\section{Búsqueda de artículos}

La búsqueda se realizó entre septiembre y noviembre de 2016 en las bases de datos Medline, Scientific Electronic Library Online (SciELO), Physiotherapy Evidence Database (PEDro), Academic Search Complete, Rehabilitation \& Sports Medicine Source y ProQuest. Fueron considerados artículos publicados entre enero de 2000 hasta noviembre de 2016.

Se utilizaron los siguientes términos descriptores médicos (Medical Subject Headings [MeSH]): Intensive Care Units, Critical Illness, Critical Care Outcomes, Patient Outcome Assessment, Outcome and Process Assessment, Validation Studies, Validity, Functional, Mobility Limitation, Physical Therapy Modalities, Physical Fitness, Physical Exertion, Physical Endurance, Motor Activity; y los operadores boleanos AND y OR.

\section{Identificación de los artículos}

Se revisaron los títulos y los resúmenes de los artículos atingentes. Luego se obtuvieron los textos extensos de aquellos resúmenes que cumplieron con los criterios de elegibilidad. Los datos fueron extraídos por 2 investigadoras (ML, PS) de manera independiente y fueron registrados en una planilla ad hoc. Se registró nombre de la escala, autor, año de publicación, tamaño de muestra, edad, género, validez de criterio y confiabilidad inter e intraobservador.

\section{Evaluación de calidad metodológica}

La calidad metodológica (CM) fue evaluada por dos revisoras (ML, PS) a través de la lista COSMIN $^{35-37}$. En este caso se consideraron los cuadros de la lista que evalúan validez de criterio y confiabilidad. Las discrepancias en las puntuaciones obtenidas entre las revisoras fueron resueltas por consenso.

\section{Síntesis de datos y análisis estadístico}

Los resultados de las variables cualitativas se expresan en porcentaje y los resultados de las variables cuantitativas se expresan en valores absolutos y rangos.

\section{Resultados}

\section{Selección de los estudios}

La búsqueda arrojó 8.526 artículos. La Figura 1 presenta el diagrama de flujo que describe la secuencia de búsqueda y razones de exclusión de artículos. Un total de 4 artículos fueron finalmente considerados para la revisión.

\section{Características de los estudios primarios}

Todos los artículos seleccionados fueron escritos en inglés y publicados entre los años 2013 y 2016. El tamaño muestral de los estudios varió entre 20 y 116 participantes, en total fueron 302 participantes, de los cuales $48,2 \%$ eran hombres y 51,8\% mujeres. Un estudio no reportó el tamaño muestral según sexo ${ }^{38,39}$. En 2 estudios se describió la edad de la muestra, cuyos promedios fueron entre 51,0 y 64,5 años ${ }^{40-43}$ (Tabla 1). De las patologías de ingreso a UPC, especificadas, las principales fueron quirúrgicas $(46,0 \%)$, seguidas por enfermedades respiratorias (17,5\%) (Tabla 2).

\section{Calidad metodológica}

Dos de los 3 artículos que evaluaban validez de criterio presentaron una CM caracterizada como "razonable". El tercero, así como los 2 que evaluaron confiabilidad, presentaron una CM categorizada como "pobre".

\section{Escalas identificadas}

Las escalas identificadas fueron: Physical Function ICU Test-scored (PFIT-s) ${ }^{42,44}$, Perme ICU Mobility Score (Perme MS) ${ }^{41}$, y De Morton Mobility Index (DEMMI) ${ }^{38}$.

\section{Validez de criterio}

En los estudios donde se evaluó la validez concurrente, se consideró al MRC-SS ${ }^{38,42-44}$ como estándar de referencia. Adicionalmente, en 1 estudio se consideró la fuerza de prensión ${ }^{42,43}$ y en otro las pruebas TUG y TM6 como estándar de referencia ${ }^{44}$. Por otro lado, en 2 estudios se evaluó la validez predictiva considerando el destino al alta ( 1 al domicilio ${ }^{44}$ y 1 a un CALP $^{42}$ ), y en 1 estudio considerando la disminución de necesidad de rehabilitación al alta hospitalaria como estándar de referencia ${ }^{44}$. 


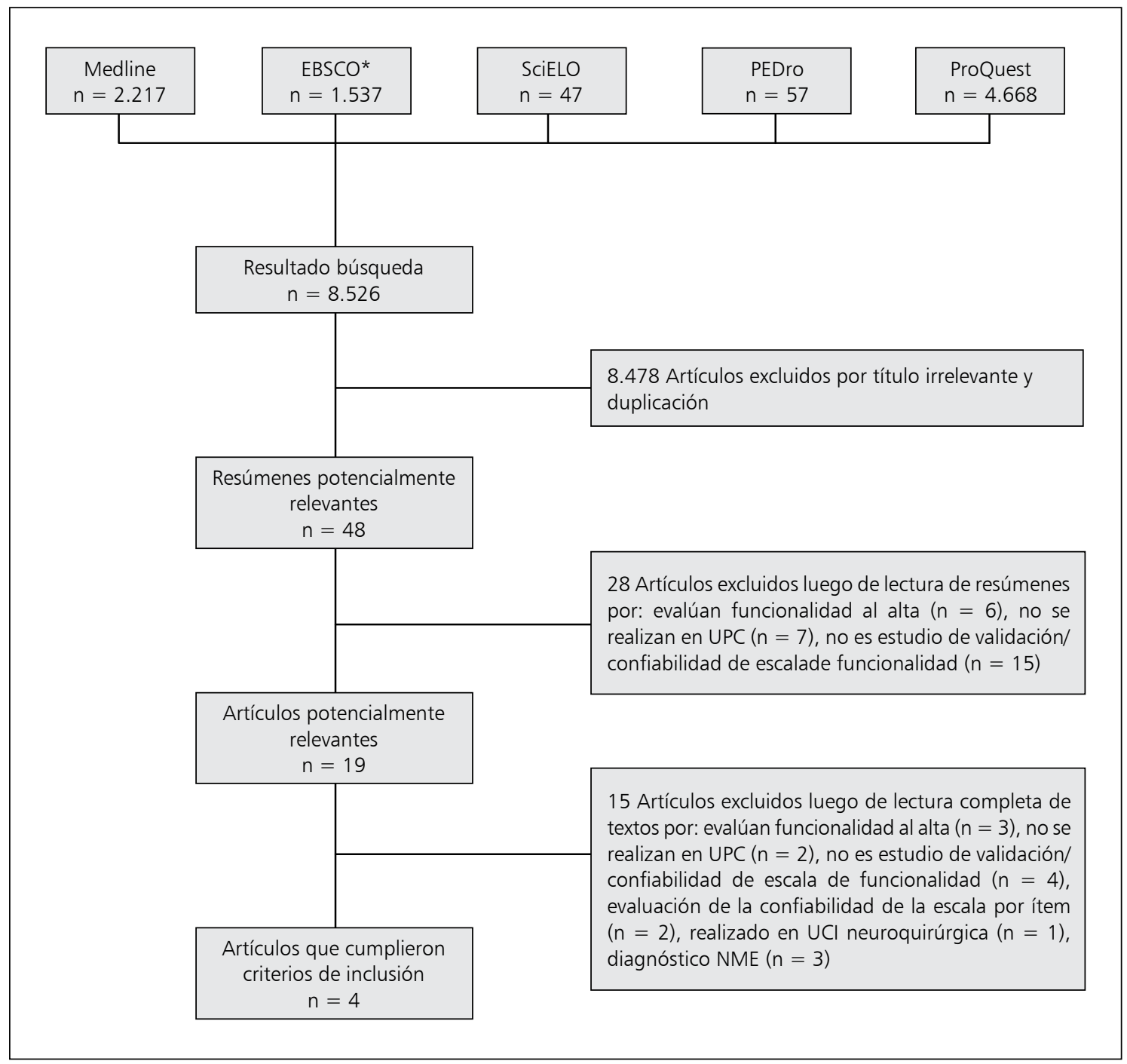

Figura 1. Diagrama de flujo.

En relación a la validez concurrente de las escalas, la escala PFIT-s presentó correlación positiva con el TM6 $(r=0,41)^{44}$, con MRC-SS (rho = entre 0,49 y 0,92$)^{42,44}$ y con la fuerza de prensión $(\text { rho }=0,76)^{45}$; así como correlación negativa con TUG $(r=-0,60)^{44}$. La escala DEMMI presentó una correlación positiva con MRC-SS ${ }^{38}$.

En relación a la validez predictiva, se observó que el puntaje de la escala PFIT-s predijo la necesidad de rehabilitación al alta $(\mathrm{OR}=0,86)^{44}$ y la probabilidad de egreso al domicilio $(\mathrm{OR}=1,20)^{44}$ (Tabla 1).

\section{Confiabilidad}

En 2 estudios fue evaluada la confiabilidad interobservador ${ }^{38,41}$, y en uno la confiabilidad intraobservador ${ }^{38}$. Todos los estudios poseen un alto nivel de confiabilidad intraobservador, reportando un CCI entre 0,93 para la escala DEMMI ${ }^{38}$ y 0,98 para la escala Perme ${ }^{41}$. Por su parte, el CCI para la confiabilidad intraobservador de la escala DEMMI fue de $0,68^{38}$.

En la Tabla 1 se muestran las propiedades de medición de cada una de las escalas identificadas. 


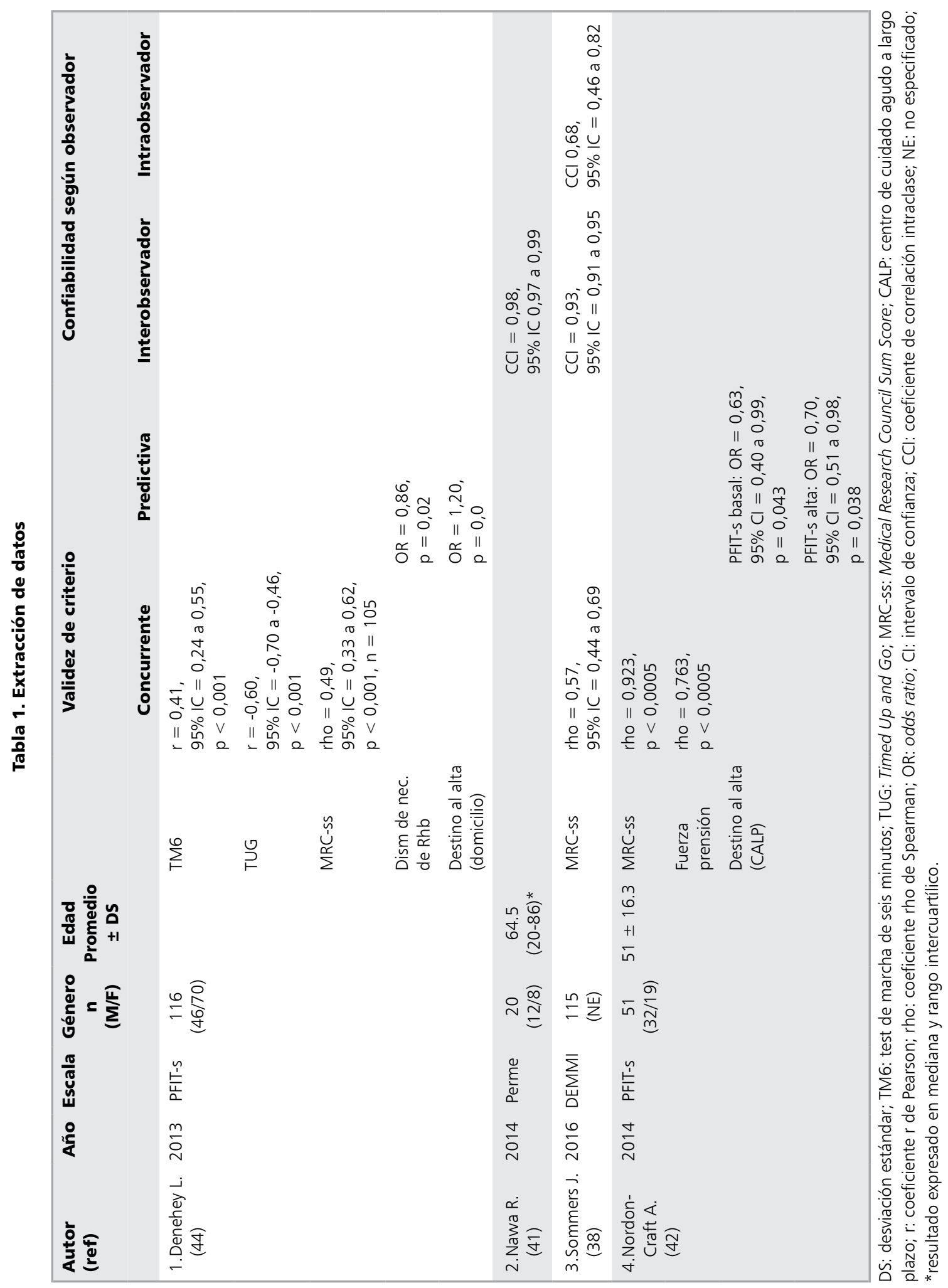


Tabla 2. Causas de ingreso a UPC

\begin{tabular}{|lrr|}
\hline Patología & n & \multicolumn{1}{c|}{$\%$} \\
\hline Respiratorio & 53 & 17,55 \\
Cardiovascular & 17 & 5,63 \\
Sepsis & 18 & 5,96 \\
Quirúrgico & 139 & 46,03 \\
Otro/no especificada & 75 & 24,83 \\
Total & 302 & 100,00 \\
\hline
\end{tabular}

aNúmero de pacientes que consideran los estudios, según diagnóstico de ingreso a UPC. Resultados expresados en valores absolutos y porcentajes.

\section{Discusión}

Este estudio fue realizado con el fin de determinar la validez de criterio y la confiabilidad inter e intraobservador de las escalas que evalúan funcionalidad en pacientes adultos críticamente enfermos. Las escalas identificadas fueron: PFIT-s, Perme MS y DEMMI. Así, los principales hallazgos de este estudio fueron que las escalas muestran diversos niveles de asociación $(0,41$ a 0,92$)$ con los estándares de referencia considerados, lo cual no permite descartar la posible influencia de variables intervinientes inadecuadamente controladas en los estudios.

La Clasificación Internacional del Funcionamiento (CIF), considera a la fuerza y masa muscular, la movilidad funcional y los factores ambientales $^{44,45}$ como factores que determinan la funcionalidad. En este contexto, todas las escalas encontradas incorporan alguno de los factores determinantes de la CIF, siendo Perme MS, la que considera más elementos en su estructura metodológica.

En una revisión sistemática (RS) recientemente publicada por Parry y cols. ${ }^{29}$ se estableció que los mejores instrumentos para evaluar fuerza, masa muscular y funcionalidad en UPC en base a sus propiedades de medición son: MRC-SS, Ultrasonografía, PFIT-s y CPAx, respectivamente. El único de estos instrumentos identificado en nuestro estudio fue la escala PFIT- $s^{42,44}$, mostrando una validez de criterio de moderada a fuerte. Vale la pena destacar que en nuestra RS, a diferencia de otras revisiones no fueron consideradas escalas de evaluación de las actividades de la vida diaria como el índice de Barthel y el de $\mathrm{Katz}^{28-32}$, las cuales incluyen en sus dominios actividades que no pueden ser realizadas en las UPC como subir y bajar escaleras, vestirse, etc ${ }^{30,31}$. Tampoco fueron incluidos estudios realizados en pacientes con patología neurológica, neuromuscular y/o traumatológica ${ }^{28,29}$, debido a que esas patologías promueven el deterioro funcional del paciente por sí mismas.

La heterogeneidad de la muestra de estudio pudiese ser un factor determinante en la variabilidad de los índices de validez calculados en los estudios primarios. En este sentido algunos estudios sugieren que el grado de severidad de la muestra pudiese ser un factor determinante en la magnitud de la asociación observada entre la escala y el estándar de referencia ${ }^{42}$.

Por otra parte, los dos estudios que analizaron la reproducibilidad de las escalas mostraron un nivel de confiabilidad moderado a alto ${ }^{38,41}$. Sin embargo, no se puede descartar que el nivel de experiencia de los evaluadores pueda afectar la confiabilidad del instrumento.

El presente estudio posee ciertas debilidades que deben ser discutidas. Así, sólo 4 estudios cumplieron con los criterios de elegibilidad. Adicionalmente, 2 de los 4 artículos encontrados presentaron una calidad metodológica categorizada como pobre y los otros 2 una calidad metodológica categorizada como razonable, por lo tanto, no es posible descartar totalmente la existencia de sesgo, pudiendo afectar a la veracidad de los resultados.

Finalmente es posible concluir que las escalas muestran una moderada validez y confiabilidad, sin embargo, debido a la variabilidad de la magnitud de asociación observada, no es posible establecer con certeza el instrumento con mejores propiedades de medición. Por este motivo, futuros estudios son necesarios para establecer los dominios que permitan determinar con mayor certeza el nivel de funcionalidad en este grupo de pacientes.

\section{Referencias}

1. Lee C, Fan E. ICU-acquired weakness: what is preventing its rehabilitation in critically ill patients? BMC Medicine 2012; 10: 115.

2. Batt J, dos Santos CC, Cameron JI, Herridge MS. Intensive Care Unit-acquired Weakness: clinical phenotypes 
and molecular mechanisms. Am J Respir Crit Care Med 2013; 187 (3): 238-46.

3. Appleton R, Kinsella J, Quasim T. The incidence of intensive care unit-acquired weakness syndromes: A systematic review. JICS 2015; 16 (2): 126-36.

4. Stevens RD, Dowdy DW, Michaels RK, Mendez-Tellez PA, Pronovost PJ, Needham DM. Neuromuscular dysfunction acquired in critical illness: A systematic review. Intesive Care Med 2007; 33 (11): 1876-91.

5. Hermans G, De Jonghe B, Bruyninckx F, Van den Berghe G. Interventions for preventing critical illness polyneuropathy and critical illness myopathy. Cochrane Database Syst Rev 2014 (1): CD006832.

6. De Jonghe B, Sharshar T, Lefaucheur JP, Authier FJ, Durand-Zaleski I, Boussarsar M, et al. Paresis Acquired in the Intensive Care Unit. A Prospective Multicenter Study. JAMA 2002; 288 (22): 2859-67.

7. de Letter MA, Schmitz PI, Visser LH, Verheul FA, Schellens RL, Op de Coul DA, et al. Risk factors for the development of polyneuropathy and myopathy in critically ill patients. Crit Care Med 2001; 29 (12): 2281-6.

8. Kress J, Hall J. ICU-Acquired Weakness and Recovery from Critical Illnes. N Engl J Med 2014; 370 (17): 162635.

9. Herridge MS, Cheung AM, Tansey CM, Matte-Martyn A, Díaz-Granados N, Al-Saidi F, et al. One-year outcomes in survivors of the acute respiratory distress syndrome. N Engl J Med 2003; 348: 683-93.

10. Alhazzani W, Alshahrani M, Jaeschke, Forel JM, Papazian L, Sevransky J, et al. Neuromuscular blocking agents in acute respiratory distress syndrome: a systematic review and meta-analysis of randomized controlled trials. Crit Care 2013; 17 (2): R43.

11. Derde S, Hermans G, Derese I, Güiza F, Hedström Y, Wouters PJ, et al. Muscle atrophy and preferential loss of myosin in prolonged critically ill patients. Crit Care Med 2012; 40 (1): 79-89.

12. Lipshutz A, Gropper M. Acquired Neuromuscular Weakness and Early Mobilization in the Intensive Care Unit. Anesthesiology 2013; 118 (1): 202-15.

13. Corner EJ, Wood H, Englebretsen C, Thomas A, Grant RL, Nikoletou D, et al. The Chelsea Critical Care Physical Assessment Tool (CPAx): validation of an innovative new tool to measure physical morbidity in the general adult critical care population; an observational proof-of. concept pilot study. Physiotherapy 2013; 99 (1): 33-41.

14. Parry S, Puthucheary Z. The impact of extended bed rest on the musculoskeletal system in the critical care environment. Extrem Physiol Med 2015; 4: 16.

15. Nordon-Craft A, Moss M, Quan D, Schenkman M. Intensive Care Unit-Acquired Weakness: Implications for Physical Therapist Management. Phys Ther 2012; 92 (12): 1494-506.

16. Corner E, Soni N, Handy JM, Brett SJ. Construct validity of the Chelsea critical care physical assessment tool: an observational study of recovery from critical illness. Crit Care 2014; 18 (2): R55.

17. Hough C, Lieu B, Caldwell S. Manual muscle strength testing of critically ill patients: feasibility and interobserver agreement. Crit Care 2014; 15 (1): R43.

18. Connolly B, Jones G, Curtis A, Murphy P, Douiri A, Hopkinson N, et al. Clinical predictive value of manual muscle strength testing during critical illness: an observational cohort study. Crit Care 2013; 17 (5): R229.

19. Latronico N, Shehu I, Seghelini E. Neuromuscular sequelae of critical illness. Curr Opin Crit Care 2005; 11 (4): 381-90.

20. Christakou A, Papadopoulos E, Patsaki I, Sidiras G, Nanas S. Functional Assessment Scales in a General Intensive Care Unit. A review. Hospital Chronicles 2013; 8 (4): 164-70.

21. Godoy D, Vaz de Mello L, Masotti L, Di Napoli M. Intensive Care Unit Acquired Weakness (ICU-AW): a brief and practical review. Reviews in Health Care 2015; 6 (1): 9-35.

22. Skinner E, Berney S, Warrillow A, Denehy L. Development of a physical function outcome measure (PFIT) and a pilot exercise training protocol for use in intensive care. Crit Care Resusc 2009; 11 (2): 110-5.

23. van der Schaaf M, Dettling DS, Beelen A, Lucas C, Dongelmans DA, Nollet F. Poor functional status immediately after discharge from an intensive care unit. Disabil Rehabil 2008; 30 (23): 1812-8.

24. Cheung AM, Tansey CM, Tomlinson G, Díaz-Granados N, Matté A, Barr A, et al. Two year outcomes, Health care use, and Costs of survivors of Acute Respiratory Distress Syndrome. Am J Crit Care Med 2006; 174 (5): 538-44.

25. van der Schaaf M, Beelen A, de Vos R. Functional outcome in patients with critical illness polyneuropathy. Disabil Rehabil 2004; 26 (20): 1189-97.

26. Poulsen J. Impaired physical function, loss of muscle mass and assessment of biomechanical properties in critical ill patients. Dan Med J 2012; 59 (11): B4544.

27. Herridge MS, Tansey CM, Matté A, Tomlinson G, Díaz-Granados N, Cooper A, et al. Functional disability 5 years after acute respiratory distress syndrome. $\mathrm{N}$ Engl J Med 2011; 364: 1293-304.

28. Parry SM, Denehy L, Beach LJ, Berney S, Williamson HC, Granger CL. Functional outcomes in ICU -what should we be using?- an observational study. Crit Care 2015; 19 (1): 127. 
29. Parry SM, Granger CL, Berney S, Jones J, Beach L, El-Ansary D, et al. Assessment of impairment and activity limitations in the critically ill: a systematic review of measurement instruments and their clinimetric properties. Intensive Care Med 2015; 41 (5): 744-62.

30. Tipping CJ, Young PJ, Romero L, Saxena MK, Dulhunty J, Hodgson CL. A systematic review of measurements of physical function in critically ill adults. Crit Care Resusc 2012; 14 (4): 302-11.

31. Christakou A, Papadopoulos E, Patsaki E, Sidiras G, Nanas S. Functional Assessment Scales in a General Intensive Care Unit. A review. Hospital Chronicles 2013; 8 (4): 164-70.

32. Elliott D, Denehy L, Berney S, Alison JA. Assessing physical function and activity for survivors of a critical illness: a review of instruments. Aust Crit Care 2011; 24 (3): 155-66.

33. Sáez E, Infante A. Guías 2004 de organización y funcionamiento de unidades de pacientes críticos. Revista Chilena de Medicina Intensiva 2004; 19 (4): 209-23.

34. Hodgson CL, Stiller K, Needham DM, Tipping CJ, Harrold M, Baldwin C, et al. Expert consensus and recommendations on safety criteria for active mobilization of mechanically ventilated critically ill adults. Crit Care 2014; 18 (6): 658.

35. Bourdin G, Barbier J, Burle JF, Durante G, Passant S, Vincent B, et al. The feasibility of early physical activity in the intensive care unit patients: A prospective observational one-center study. Respir Care 2010; 55 (4): 400-7.

36. Mokkink L, Terwee C, Patrick D, Alonso J, Stratford P, Knol D, et al. COSMIN checklist manual. 2012.

37. Terwee CB. COSMIN checklist with 4-point scale.

38. Sommers J, Vredeveld T, Lindeboom R, Nollet F, Engelbert RH, van der Schaaf M. de Morton Mobility Index Is Feasible, Reliable, and Valid in Critically Ill Patients. Phys Ther 2016; 96 (10): 1658-66.

39. Corner E, Wood H, Englebretsen C, Thomas A, Grant RL, Nikoletou D, et al. The Chelsea Critical Care Physical Assessment Tool (CPAx): validation of an inno- vative new tool to measure physical morbidity in the general adult critical care population; an observational proof-of-concept pilot study. Physiotherapy 2013; 99 (1): 33-41.

40. Hodgson A, Needham D, Haines K, Bailey M, Ward A, Harrold M, et al. Feasibility and inter-rater reliability of the ICU Mobility Scale. Heart Lung 2014; 43 (1): 19-24.

41. Nawa RK, Lettvin C, Winkelman C, Evora PR, Perme C. Initial interrater reliability for a novel measure of patient mobility in a cardiovascular intensive care unit. J Crit Care 2014; 29 (3): 475.e1-5.

42. Nordon-Craft A, Schenkman M, Edbrooke L, Malone DJ, Moss M, Denehy L. The Physical Function Intensive Care Test: Implementation in Survivors of Critical Illness. Phys Ther 2014; 94 (10): 1499-507.

43. Huang M, Chan K, Zanni J, Parry SM, Neto SG, Neto JA, et al. Functional Status Score for the ICU: An International Clinimetric Analysis of Validity, Responsiveness, and Minimal Important Difference. Crit Care Med 2016; 44 (12): e1155-64.

44. Denehy L, de Morton NA, Skinner EH, Edbrooke L, Haines K, Warrillow S, et al. A Physical Function Test for Use in the Intensive Care Unit: Validity, Responsiveness, and Predictive Utility of the Physical Function ICU Test (Scored). Phys Ther 2013; 93 (12): 1636-45.

45. World Health Organization. International Classification of Functioning, Disability and Health. WHO, Geneva. 2001.

46. Perme C, Kenji Nawa R, Winkelman C, Faisal Masud. A tool to assess mobility status in critically ill patients: The Perme Intensive Care Unit Mobility Score. Methodist Debakey Cardiovasc J 2014;10 (1): 41-9.

47. Castro A, Serón P, Fan E, Gaete M, Mickan S. Effect of Early Rehabilitation during Intensive Care Unit Stay on Functional Status: Systematic Review and Meta-Analysis. PLos One 2015; 10 (7): e0130722.

48. Connolly B, Salisbury L, O’Neill B, Geneen L, Douiri A, Grocott MP, et al. Exercise rehabilitation following intensive care unit discharge for recovery from critical illness. Cochrane Database Syst Rev 2015; 6: CD008632. 